\title{
Influence of Composition on Properties of Hydrogels of 2-Hydroxyethyl Methacrylate with a Sulphobetaine Comonomer
}

\author{
Jose M. ReGo* and Malcolm B. Huglin \\ Department of Chemistry and Applied Chemistry, University of Salford, \\ Salford M5 $4 W T, U K$.
}

(Received February 22, 1991)

\begin{abstract}
The copolymerization of 2-hydroxyethyl methacrylate (HEMA) and a zwitterionic methacrylate $N, N$-dimethyl- $N$-methacryloxyethyl- $N$-(3-sulphopropyl)-ammonium betaine in the presence of a tetrafunctional crosslinker has been effected to $100 \%$ conversion by $\gamma$-irradiation. The resultant xerogels were swollen to equilibrium in water to yield hydrogels. The water uptake, types of water and the mechanical properties were examined and discussed with relation to the composition of the copolymer. The synergistic swelling behaviour found has been explained on the basis of calculated microstructural composition.

KEY WORDS 2-Hydroxyethyl Methacrylate / Zwitterionic Methacrylate / Copolymer / Hydrogel / Swelling / Microstructure /
\end{abstract}

In recent papers,,${ }^{1,2}$ the influence of temperature $T$ and the presence of the salt potassium thiocyanate KCNS in the swelling medium on the properties of a hydrogel based on the zwitterionic monomer $N, N$-dimethyl- $N$-methacryloxyethyl- $N$-(3-sulphopropyl)ammonium betaine (SPE) were reported. These studies showed that PSPE hydrogels exhibit interesting swelling properties, viz., (i) a degree of gravimetric equilibrium swelling in water $W$ of 0.623; (ii) a sharp increase in $W$ and decrease in the volume fraction of polymer $\phi_{2}$ in aqueous $\mathrm{KCNS}$ as swelling medium; (iii) an interaction parameter $\chi$ of 0.67 in water and 0.49 in aqueous KCNS which is independent of salt concentration; (iv) a low Young's modulus $E$ in water and aqueous $\mathrm{KCNS}$; (v) a swelling mechanism in water which is endothermic at $T<353 \mathrm{~K}$ and exothermic at higher temperatures: (vi) a negative entropy of dilution in water which controls the swelling process at high $T$.
These properties mentioned were ascribed to the presence of the zwitterionic group in SPE. In these studies, however, the relative importance of the sulphobetaine possible couplings, viz., intra-group associations, interactions between nearest neighbours or intra-chain or inter-chain coupling was not elucidated. This could be attained by introducing neutral spacers between SPE monomer units which would allow one to at least evaluate the importance of nearest neighbours interactions. Introduction of spacers is possible by copolymerizing SPE with a suitable monomer.

This work is aimed at determining the changes is swelling and network properties as a function of SPE composition within the xerogel for hydrogels based on 2-hydroxyethyl methacrylate (HEMA) and SPE using ethyleneglycol dimethacrylate (EDMA) as crosslinker. The selection of HEMA as comonomer for SPE was dictated by the following considerations: (i) the wide variety of industrial

* Present address: UEE Explosivos, Fca Galdacano, Departamento de I \& D, Apdo de Correos 6, 48960 Galdacano, Vizcaya, Spain. 
applications $^{3-6}$ for which PHEMA is appropriate; (ii) the moderate Young's modulus E exhibited by PHEMA hydrogels which ranges ${ }^{7}$ between 0.5 and $1.2 \mathrm{MN} \mathrm{m}^{-2}$ and (iii) the moderate water uptake due to the presence of the hydrophilic hydroxyl group. Accordingly, the copolymerization of these two monomers is expected to produce hydrogels having $W$ values which vary between 0.39 and 0.623 and a modulus $E$ ranging from 0.037 to $0.6 \mathrm{MN} \mathrm{m}^{-2}$ (values found for PSPE and PHEMA hydrogels respectively).

It should be noted, furthermore, that this study is not only interesting from an academic viewpoint but it also presents a high industrial potential since the copolymerization studied can be understood as a modification of PHEMA hydrogels, rather than an improvement of PSPE gels, to produce materials with new properties.

\section{EXPERIMENTAL PROCEDURE}

\section{Materials.}

SPE (Rasching Co.) and EDMA (Aldrich Chem. Co.), HEMA (Ubichem Ltd., 99.7 wt \% pure; EDMA content $=0.08 \mathrm{wt} \%$ ) and ammonium persulphate (Aldrich Chemical Co.) were used as received. Doubly distilled deionized water was used for polymerization, swelling and stress-strain experiments.

\section{Preparation of Low Conversion Copolymers}

Aqueous solutions of SPE (1), HEMA (2) and the initiator ammonium persulphate were outgassed with gaseous nitrogen for $30 \mathrm{~min}$ prior to the polymerization at $323 \mathrm{~K}$. The initial total concentrations of monomers and initiator were $25 \mathrm{~g} \mathrm{dm}^{-3}$ and $2 \times 10^{-3} \mathrm{~mol} \mathrm{dm}^{-3} \mathrm{re}-$ spectively. Unreacted monomers were removed by dialysis with deionized water for 3 days. In order to enhance accuracy of determination of copolymer composition, mole fractions of SPE in the feed $\left(f_{1}\right)$ higher than 0.3 were prepared. The polymerization time range between $50 \mathrm{~min}$ for the lowest $f_{1}(0.4)$ and $150 \mathrm{~min}$ for the highest $f_{1}(0.95)$. under these conditions conversions in the range $3-10 \mathrm{wt} \%$ were attained. The copolymers were obtained as thin films after evaporation of water in an oven at $348 \mathrm{~K}$ and final drying in a vacuum oven at $314 \mathrm{~K}$ for $48 \mathrm{~h}$.

\section{Copolymer Analysis and Reactivity Ratio De- termination}

In principle, Kjeldahl nitrogen analysis is suitable for copolymer analysis, since nitrogen is present in SPE but not in HEMA. However, the maximum nitrogen content (in polySPE (PSPE)) is relatively small, 6.9 wt\%. Also, militating against the adoption of this method was the surprising experimental observation that it yielded $0 \%$ for these copolymers. This is presumably because a salt is formed which cannot be broken down to ammonia. Accordingly, analysis was made on the basis of content of sulphur, the maximum possible amount being $11.5 \mathrm{wt} \%$ in PSPE. Determinations were made by Butterworth Laboratories Ltd. (Teddington, Middlesex, UK) by means of an oxidation procedure followed by dionex ion exchange chromatography. The feed and copolymer composition data were analyzed by using the Fineman-Ross method. ${ }^{8}$

\section{Preparation of Hydrogels}

Aqueous solutions of SPE, HEMA, and EDMA were prepared gravimetrically. Five SPE feed mole fractions were made up, viz., $0.15,0.30,0.45,0.60$, and 0.75 . In every preparation, the EDMA concentration used was $0.5 \mathrm{wt} \%$. Every feed composition required a different water content in order to compensate for two opposite effects: (i) when the water content is too high ( $>c a .40 \%$ ) PHEMA phase separates and (ii) when the water content is too low $(<c a .50 \%)$ SPE tends to gel. ${ }^{9}$ The following volumes of water per gram of monomer mixture were found to be more convenient in order to compensate for the effects mentioned: $0.4,0.5,0.8,1.0$ and $1.2 \mathrm{~cm}^{3}$ for $f_{1}=0.15,0.30, \quad 0.45, \quad 060$, and 0.75 
respectively. The reaction mixtures were outgassed with gaseous nitrogen and irradiated with $\gamma$-irradiation from a $9000 \mathrm{Ci}{ }^{60} \mathrm{Co}$ source. The $\gamma$-irradiation dose was $1 \mathrm{M} \mathrm{rad}$, the dose rate being $0.01 \mathrm{M} \mathrm{rad} \cdot \mathrm{h}^{-1}$ as determined by Fricke dosimetry. The resultant hydrogel rods were swollen to equilibrium for two months. During this time the unreacted monomer was removed by changing the swelling medium once a day. Parallel to this swelling conditioning to equilibrium of the hydrogels, pre-weighed dry fragments of them were Soxhlet extracted for $24 \mathrm{~h}$ in hot water. After Soxhlet extraction, the gel fragments were dried in an oven at $348 \mathrm{~K}$ during $48 \mathrm{~h}$ followed by subsequent drying in a vacuum oven during another $48 \mathrm{~h}$. The weight of the fragments prior to the Soxhlet extraction and after the extraction were found to be the same. Consequently, $100 \mathrm{wt} \%$ conversion was attained in all cases. The feed compositions, therefore, are equal to the overall copolymer compositions, denoted as the mole fraction $F_{1}$ of SPE in the copolymer. Hydrogels from PSPE and PHEMA crosslinked with EDMA were also prepared as described in ref. 2.

After swelling equilibrium was reached, the rods were inserted in metal tubes of appropriate thickness and diameter and the protruding end was cut with a scalpel to produce thin discs (diameter $\approx 11 \mathrm{~mm}$ and thickness $\approx 2 \mathrm{~mm}$ ) for measurement of swelling and cylindrical pellets (diameter $\approx 11 \mathrm{~mm}$ and thickness $\approx 11 \mathrm{~mm}$ ) for measurements of compression modulus.

\section{Equilibrium Water Content of Hydrogels}

The equilibrium water content of the hydrogels $W$ was determined gravimetrically (ratio of the mass of water within the hydrogel to the total mass of hydrogel). Dry weighed xerogel discs were equilibrated in deionized water at $293 \mathrm{~K}$ for two weeks. Since the xerogel discs used here were previously subjected to the procedure described before to remove unreacted monomer, the $W$ values given here are true ones. The reproducibility was tested by measuring $W$ for six different discs. The standard deviation found for $W$ was 0.01 .

\section{Volumetric Swelling}

The volume fraction of polymer within a hydrogel $\phi_{2}$ is given, at a particular temperature, by

$$
\phi_{2}=\left(D_{0} / D\right)^{3}
$$

where $D_{0}$ and $D$ are the diameters of dry and swollen discs respectively. $D_{0}$ was measured at $293 \mathrm{~K}$ with a micrometer. A Minolta X300 35 $\mathrm{mm}$ camera fitted with fitted with a Tamron $90 \mathrm{~mm} f / 2.5$ lens was used in the photographic procedure, details of which have been given previously. ${ }^{10}$

\section{Compression Measurememts}

Elastic moduli of the hydrogels were determined by stress (compression)-strain experiments at $293 \mathrm{~K}$. Full experimental details of the assembly used are given elsewhere. ${ }^{10}$

\section{Differential Scanning Calorimetry (DSC)}

The d.s.c. attachment to the Mettler thermal analyser (model TA 3000) was used. Surfacedried hydrogel $(\approx 4-20 \mathrm{mg})$ was placed in a DSC pan, which was sealed. The temperature was reduced to $243 \mathrm{~K}$ and then raised to $298 \mathrm{~K}$ at a heating rate of $2.5 \mathrm{~K} \mathrm{~min}^{-1}$. A peak corresponding to the melting endotherm of water occurred between 263 and $283 \mathrm{~K}$, the peak maximum being located between 269 and $278 \mathrm{~K}$. The breadth of the peak depended on the content of normal freezing water present in the sample. The mass of freezing water was obtained by integrating of the peak area, using facilities attached to the instrument, in conjunction with the enthalpy of fusion of pure water, $\Delta H_{\mathrm{f}}$. Using pure water alone a value of $\Delta H_{\mathrm{f}}=329.2 \mathrm{~J} \mathrm{~g}^{-1}$ was obtained as the average of 12 determinations each involving a different mass of water in the pan. The mass of non-freezing water in the hydrogel was obtained as the difference between the known total equilibrium water content and the mass 
of freezing water. Expressed as a fraction of the total weight of water, the contents of freezing and non-freezing water are denoted by $W_{\mathrm{f}}$ and $W_{\text {nf }}$ respectively.

\section{RESULTS AND DISCUSSION}

\section{Monomer Reactivity Ratios}

Values of the monomer reactivity ratios $r_{1}$ and $r_{2}$ were determined as to be $0.59 \pm 0.04$ and $1.14 \pm 0.08$ respectively.

\section{Swelling Properties}

The gravimetric water content $W$ as a function of $F_{1}$ is given in Figure 1(a), and Figure 1(b) shows the variation of the freezing water content $W_{\mathrm{f}}$ with $F_{1}$. The main feature of Figure 1a is the existence of a maximum in $W$ for $0.45<F_{1}<0.60$. $W_{\mathrm{f}}$ shows a similar variation with $F_{1}$ to that observed for $W$ and exhibits a maximum in the same range. It should also be noted that the degree of swelling observed is higher than that expected on the basis of additivity of swelling of the copolymers with respect to the hydrogels formed from the homopolymers (dashed line). Hence, the swelling of SPE/HEMA hydrogels as a function of $F_{1}$ is synergistic.

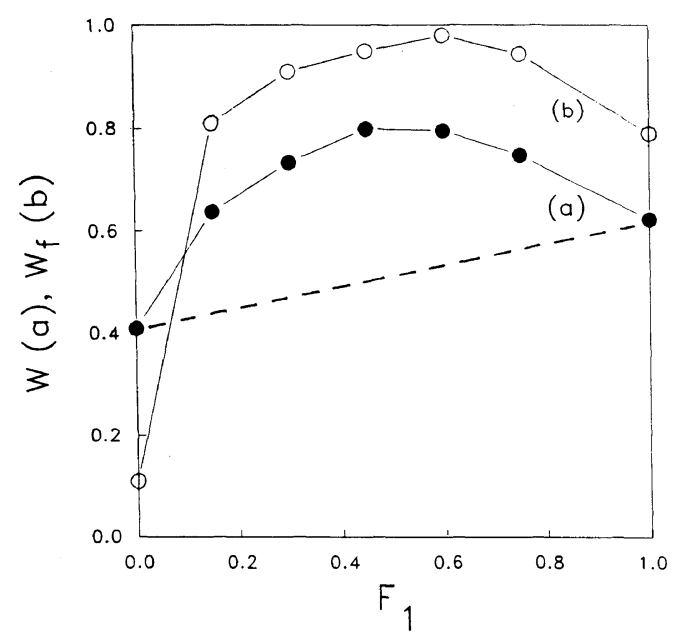

Figure 1. Variation of (a) the gravimetric water content $W$ and (b) the freezing water content with mole fraction $F_{1}$ of SPE in copolymer.
In a previous paper ${ }^{11}$ on the swelling behaviour of copolymeric hydrogels containing $N$-vinyl-2-pyrrolidone (VP), the representation of the mole fraction of VP units within the hydrogel as a function of the mole fraction of water molecules in the hydrogel $X_{\mathrm{w}}$ disclosed a common trend among copolymeric hydrogels comprising a hydrophilic monomer (VP) and different hydrophobic ones, viz. the existence of a maximum due to a sharp increase in water content of the freezing or bulk type. Figure 2, which gives the mole fraction of SPE units in the hydrogel $X_{1}$ as a function of $X_{\mathrm{w}}$ for copolymeric hydrogels based on SPE and HEMA, does not exhibit a maximum. Instead, there are two regimes of swelling behaviour: (i) between $X_{\mathrm{w}}=0.834 \quad\left(F_{1}=0\right)$ to $X_{w}=0.978$ $\left(F_{1}=0.45\right)$, the hydrogels behave in a similar manner to that found for hydrogels containing VP, viz., an increase of water content of hydrogel with content of the more hydrophilic monomer, and (ii) from $X_{\mathrm{w}}=0.978\left(F_{1}=0.45\right)$ to $X_{\mathrm{w}}=0.962\left(F_{1}=1\right), X_{\mathrm{w}}$ decreases as $X_{1}$ increases. Discussion of these two regimes will be made later in the text.

The calorimetric data on freezing and non-freezing water have been used to calculate the ratios $W_{\mathrm{f}} / W_{\mathrm{nf}}$ for the copolymeric

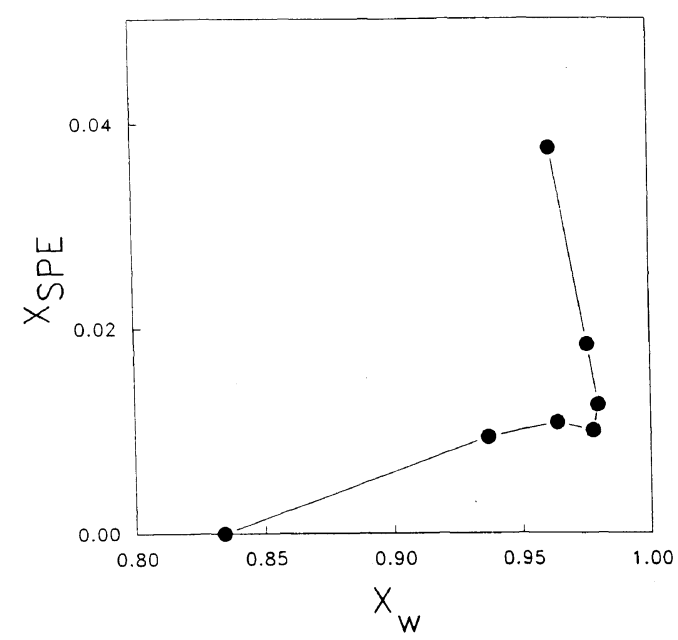

Figure 2. Variation of mole fraction of SPE units within the hydrogel $X_{\text {SPE }}$ with mole fraction of water inside the gel $\mathrm{X}_{\mathrm{w}}$. 
Table I. Ratio of freezing to non-freezing water in SPE/HEMA hydrogels from xerogels of different mole fractions $F_{1}$ of SPE

\begin{tabular}{llc}
\hline$F_{1}$ & $W_{\text {nf }}$ & $W_{\mathbf{f}} / W_{\text {nf }}$ \\
\hline 0.0 & 0.89 & 0.1 \\
0.15 & 0.19 & 4.3 \\
0.30 & 0.09 & 10.1 \\
0.45 & 0.05 & 19.0 \\
0.60 & 0.02 & 49.0 \\
0.75 & 0.056 & 16.9 \\
1.0 & 0.21 & 3.8
\end{tabular}

hydrogels of different composition which are given in Table I. It is seen from this table that freezing water predominates over non-freezing water over most of the composition range and this effect is most pronounced between $F_{1}=0.45$ and $F_{1}=0.75$.

Summarising, copolymeric hydrogels comprising HEMA and SPE exhibit a unique swelling behaviour mainly characterized by: (i) a maximum for the curve $W v s . F_{1}$. The profile of this curve, moreover, is very dissimilar to the expected additive behaviour (synergistic swelling) and (ii) high concentration of freezing water. Comparison of the swelling behaviour found for these types of hydrogel with the one reported for VP/HEMA hydrogels ${ }^{11}$ suggests that this novel swelling behaviour must be ascribed to SPE.

The zwitterionic group in SPE could give rise to a variety of possible interactions which can affect the hydrogel swelling behaviour as was shown recently. ${ }^{1,2)}$ In this connection, the coupling of the charged groups $-\mathrm{SO}_{3}^{-}$and $-\mathrm{N}^{+}\left(\mathrm{CH}_{3}\right)_{2}-$ with salts and subsequent increase in water uptake reveals the influence of ionic interactions on the overall swellability of the gel. The intra-group and intra-chain associations appear to be of relevance as pointed out elsewhere. ${ }^{9}$ With respect to the latter, the interactions between nearest neighbours are especially amenable to being studied via determination of the concentration of likeunlike dyads within the gel as a function of $F_{1}$.

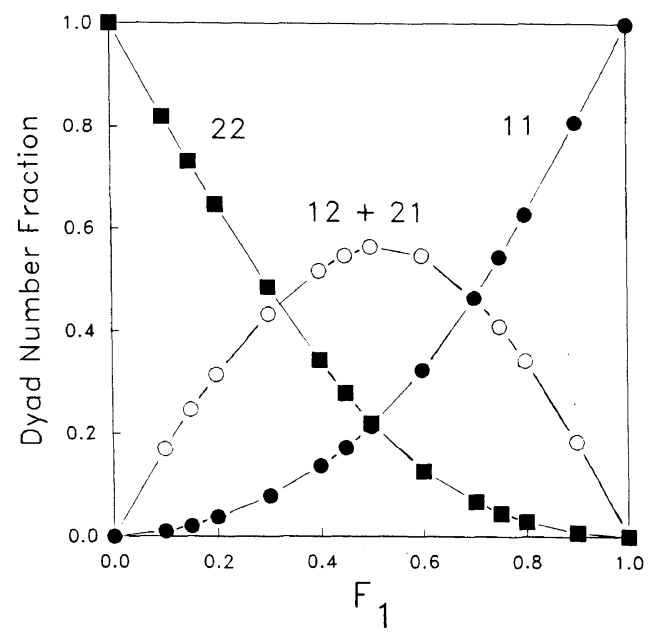

Figure 3. Dyad distribution as a function of $F_{1}$ obtained by means of a Monte Carlo simulation of copolymerization. Input data: total number of feed molecules $=50000$; conversion $=100 \% ; r_{1}=0.59$ and $r_{2}=1.14$.

This structural study can be undertaken by measuring suitable NMR peaks or by using a Monte Carlo simulation method under the assumption of a first order Markov process. A computer programme has been developed in our research group which calculates different types of sequence length distribution (SLD) using the Monte Carlo simulation method. This simulation has been found to provide suitable SLD data when the initial propagation probability pairs $\left(P_{11}, P_{22}\right)$ are not very dissimilar. Further and extensive details of the simulation and the computer programme are given in ref 12 . The reactivity ratios found here for SPE (1)/HEMA (2) copolymers in conjunction with the values of $F_{1}(0.15-0.75)$ and the extremely high conversions attained, provide conditions that have been shown to be optimum for obtaining reliable SLD data ${ }^{12}$ from the simulation. The results obtained are given in Figure 3 in the form of mole fractions of dyads of like-like monomer units (11 and $22)$ and like-unlike monomer units $(12+21)$ as a function of xerogel composition $F_{1}$. As seen from this figure, the number of like-unlike dyads passes through a maximum located between $F_{1}=0.4$ and $F_{1}=0.7$. HEMA-HEMA 
dyads (22) are dominant before the maximum and SPE-SPE dyads (11) after the maximum. The maximum in water uptake indicated in Figure 1 thus coincides with a maximum of like-unlike dyads. Similarly, the two regimes of swelling behaviour in Figure 2 correspond to copolymers having microstructures in which dyads of the type HEMA-HEMA and HEMS-SPE are dominant in the first regime and those of the type SPE-SPE are dominant in the second regime. Thus, it appears that replacement of one of the SPE units in a SPE-SPE dyad by a different nearest neighbour reduces the intra-group association within the zwitterionic monomer unit and allows a greater ingress of water than occurs without such replacement. This finding is significant with regard to the observed synergistic swelling behaviour exhibited by this class of copolymers. We are unable to quantify this, but any possible mechanism should account for some form of electrostatic interaction within SPESPE dyads which is significantly reduced within SPE-HEMA dyads.

\section{Network Parameters}

Young's moduli $E$ were obtained as the slopes in plots of stress $\tau v s$. strain $(\lambda-1)$. Here $\tau$ is the applied force per unit area of hydrogel and $\lambda$ is the ratio of deformed length to undeformed length of hydrogel. Over the range of strain covered, 0 to -0.07 , the plots were linear.

The theoretical crosslinking density $v_{\mathrm{t}}$ has been calculated from

$$
v_{\mathrm{t}}=\frac{C f}{2}
$$

where $C\left(\mathrm{moldm}^{-3}\right)$ is the concentration of crosslinking agent of functionality $f$. For EDMA $f=4$. The value of $C$ was obtained from the weight concentration of EDMA and by taking the density $\rho$ of the xerogels as $1.10 \mathrm{~kg} \mathrm{dm}^{-3}$. The latter was determined by direct weighing and micrometrically measured dimensions of the dried discs and pellets used.
The effective crosslinking density was calculated experimentally from the compressionstrain $^{11}$ results via eq 3 and 4 in which $G$ is the compression modulus, $R$ is the gas constant, $T$ $(\mathrm{K})$ is the temperature and $v_{\mathrm{e}}$ is the effective crosslinking density:

$$
\begin{gathered}
\tau=G\left(\lambda-\lambda^{-2}\right) \\
G=R T v_{\mathrm{e}} \phi_{2}^{1 / 3}
\end{gathered}
$$

At low strains, i.e., 0 to about -0.07 , plots of $\tau$ vs. $\left(\lambda-\lambda^{-2}\right)$ according to eq 3 were linear, thereby yielding the compression moduli $G$ as the slopes from which values of $v_{\mathrm{e}}$ were isolated via eq 4. Values of the polymer-water interaction parameter $\chi$ were calculated from the following expression valid at swelling equilibrium ${ }^{13}$ :

$$
\phi_{2}^{2}(0.5-\chi)-V_{1}\left[v_{\mathrm{e}} \phi_{2}^{1 / 3}-\left(2 v_{\mathrm{e}} / f\right) \phi_{2}\right]=0
$$

In eq 5 the water molar volume $V_{1}$ at $293 \mathrm{~K}$ is equal to $18 \times 10^{-3} \mathrm{dm}^{3} \mathrm{~mol}^{-1}$.

The Young's modulus $E$ and the compression modulus $G$ for rubbery materials are related through the following equation (eq 14):

$$
E=k G=k R T v_{\mathrm{e}} \phi_{2}^{1 / 3}
$$

The validity of eq 6 is illustrated in Figure 4, where the plot of $E v s . \phi_{2}^{1 / 3} v_{\mathrm{e}}$ is seen to be linear and to pass through the origin. The data points

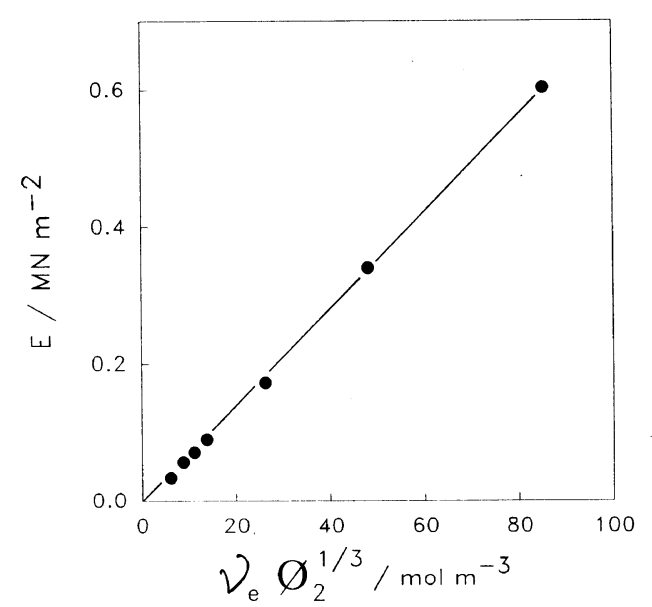

Figure 4. Dependence of Young's modulus on $\phi_{2}^{1 / 3} v_{\mathrm{c}}$ for the polymeric and copolymeric hydrogels. 


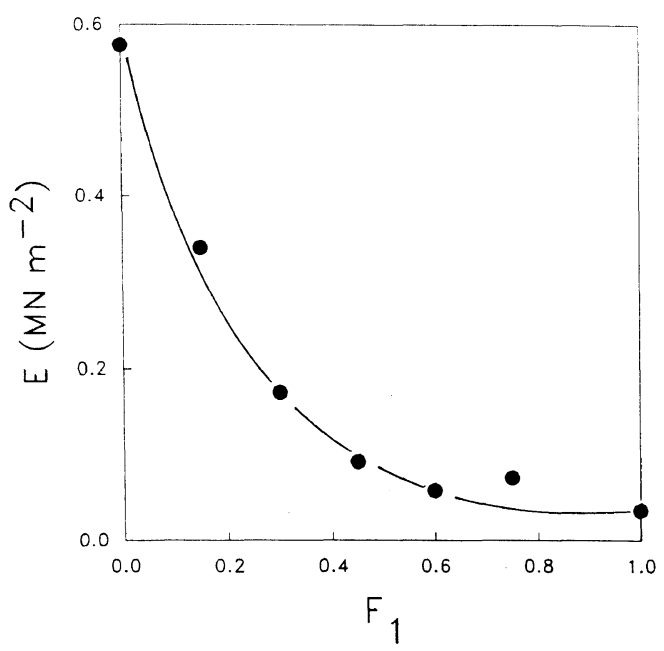

Figure 5. Change of Young's modulus of hydrogel with composition of unswollen copolymer.

relate to the hydrogels of PHEMA, PSPE and of the copolymers of five different compositions. The slope, in conjunction with $R=8.31$ $\mathrm{J} \mathrm{K}^{-1} \mathrm{~mol}^{-1}$ and $T=293 \mathrm{~K}$, affords a value of $k=2.95$, which accords well with the value of 3 predicted for the ratio $E / G$ in the limit of very small strain. ${ }^{14}$

For the same systems, Figure 5 gives the variation of Young's modulus of hydrogel with composition of the unswollen gel. It will be convenient to consider the latter as two regimes, viz., region $1\left(F_{1}=0\right.$ to 0.6$)$ and region $2\left(F_{1}=0.6\right.$ to 1$)$. Within region $1, E$ undergoes a very sharp decrease, which is compatible with and expected from increases in swelling ( $c f$. Figure 1) and the values of $W_{\mathrm{f}} / W_{\mathrm{nf}}(c f$. Table I) in the same regime. Moreover, the density of xerogels $\left(1100 \mathrm{~kg} \mathrm{~m}^{-3}\right)$ allows the theoretical crosslinking density to be calculated via. eq 2 ; this yields $v_{\mathrm{t}}=56 \mathrm{~mol} \mathrm{dm}^{-3}$ for all samples. However, the effective crosslinking density changes with composition. Thus, for $F_{1}=0$, $0.15,0.30,0.45,0.60,0.75$, and 1.0 , the values of $v_{\mathrm{e}}$ are $101,67,41,24.5,15,18$, and 8.6 moldm ${ }^{-3}$, respectively. Hence within region 1 there is also a sharp decrease in $v_{\mathrm{e}}$.

Within region 2 the opposite situation prevails with regard to the values of $W_{\mathbf{f}} / W_{\mathbf{n f}}$ and of the degree of swelling, since both quantities undergo a decrease in value. Also the falls in $E$ and $v_{\mathrm{e}}$ are only very small relative to those in region 1. It is relevant to note that non-freezing or bound water can make a positive contribution to effective crosslinking and this may account for the finding that $v_{\mathrm{e}}$ does not decrease markedly in this region 2 .

However, the overall decreasing effectiveness of the crosslinker EDMA with increase in $F_{1}$ can be assessed quantitatively by considering the actual formation of the xerogels by the terpolymerization process in which the reactivity ratios (or the $Q, e$ values) of the monomers play a critical role. This will now be examined in more detail. It should be recalled that this regime (2) of elasticity corresponds to the range of copolymer composition for which SPE-SPE dyads are predominant $\left(F_{1}>0.6\right)$ (Figure 3$)$. Therefore, copolymers showing a structure comprised primarily of SPE-SPE dyads, and thereby showing strong electrostatic interactions between nearest neighbours, exhibit a different behaviour from copolymers consisting mainly of HEMA-HEMA and SPE-HEMA dyads $\left(F_{1}<0.6\right)$.

One possible way to monitor the changes in the instantaneous composition of SPE and crosslinker in the copolymer $\left(F_{1}\right.$ and $F_{3}$ respectively) and, accordingly the homogeneity of crosslinking, was given in a previous article. ${ }^{15}$ In brief, this method establishes that the instantaneous copolymer composition of the component $h\left(F_{\mathrm{h}}\right)$ of a ternary system monomer/monomer/crosslinker can be expressed as:

$$
F_{\mathrm{h}}=\frac{f_{\mathrm{h}} Q_{\mathrm{h}}\left[\sum f_{i} Q_{i} \exp \left(-e_{\mathrm{h}} e_{i}\right)\right]}{\sum \sum\left[f_{i} Q_{i} f_{j} Q_{j} \exp \left(-e_{i} e_{j}\right)\right]}
$$

In this equation $f_{i}$ is the mole fraction of the component $i$ in the feed and $Q_{i}$ and $e_{i}$ are the general reactivity constant and general polarity of the component $i$ respectively. The denominator in eq 7 corresponds to the consumption of all monomers and is therefore the same for $F_{1}$, 
$F_{2}$, and $F_{3}$. The numerator, however, is specific of each monomer and defines the incorporation of each one into the polymer. If the $Q_{i}$ and $e_{i}$ values as well as the initial feed compositions $f_{i}^{0}$ are known for all monomers involved, $F_{i}$ can be obtained via eq 7 at the onset of polymerization. However, if instantaneous copolymer compositions are required throughout the range of conversion, it is necessary to specify the interval of conversion during which the composition $F_{1}$ is attained. The smaller the interval taken, the more accurate will be the predicted drift in composition. Thus, on the assumption that $1 \%$ of the monomer originally present is converted to polymer of the predicted composition, an overall mass balance can be calculated:

$$
F_{i}=\sum F_{i} / x
$$

In eq $8, F_{i}$ is the mole fraction of monomer $i$ in the accumulated polymer (i.e., the average polymer composition) at the interval $x$ of conversion, which is the percentage conversion in this case. After each interval of conversion, the composition of unreacted monomer feed $f_{i}$ can be calculated from the following expression in which $\theta$ denotes the fractional conversion of all monomers:

$$
f_{i}=\left(f_{i}^{0}-F_{i} \theta\right) /(1-\theta)
$$

This material balance yields new values for $f_{i}$ after each interval of conversion. By repeating this sequence of procedures 100 times, for example, a graphical representation of polymerization can be made. This method is applicable for any number $(n)$ of monomers. Here a programme was written in BASIC for $n=3$. The output was in tabulated form. The $Q$ and $e$ values adopted for the three monomers were the following: $\mathrm{SPE}^{16}, Q=1.59$, $e=1.74 ;$ HEMA $^{16}, Q=2.8, \quad e=1.12$, and EDMA $^{17}, Q=0.88$ and $e=0.24$.

Theoretical plots for the terpolymerization SPE (1)/HEMA (2)/EDMA (3) of fractional conversion $\theta$ vs. $F_{1}$ and $F_{3}$ (Figures 6 and 7 , respectively) for different mole fraction feed

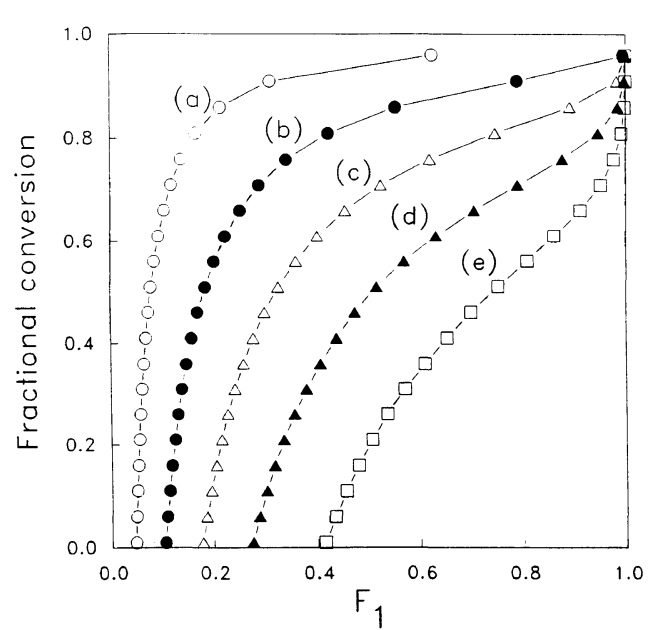

Figure 6. Fractional conversion $\theta$ as a function of instantaneous copolymer composition in SPE $F_{1}$. (a), (b), (c), (d), and (e) correspond to initial feed compositions of monomer 1 (SPE) $f_{1}^{0}=0.15,0.30,0.45,0.60$, and 0.75 , respectively.

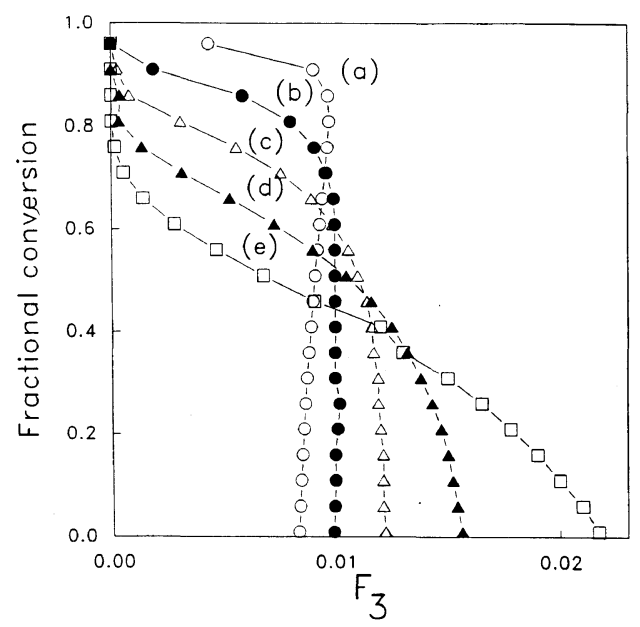

Figure 7. Fractional conversion $\theta$ as a function of instantaneous copolymer composition in EDMA $F_{3}$. (a), (b), (c), (d), and (e) correspond to intial feed compositions of monomer 1 (SPE) $f_{1}^{0}=0.15,0.30,0.45,0.60$ and 0.75 , respectively.

compositions indicate that: (i) the copolymer is primarily comprised of HEMA units at low conversions. The SPE compositional drift, observed at different conversions depending on the initial feed composition, must be attributed to the fact that the feed mixture must be 
richer in SPE at medium to high $\theta$ due to the more favourable consumption of HEMA during the earlier stages of the copolymerization: (ii) EDMA tends to react with HEMA radicals and accordingly a more homogeneous distribution of crosslinking is obtained the lower the initial SPE composition. Thus, the EDMA compositional drift appears at $\theta \approx 0.9$, $0.75,0.6,0.4$, and 0.2 for $F_{1}=0.15,0.3,0.45$, 0.6 , and 0.75 , respectively.

It should be noted that, although the initial EDMA content of $0.5 \mathrm{wt} \%$ corresponds to a $f_{3}^{0}=0.0043$, for the calculations $f_{3}^{0}$ was taken as 0.0086 to account for the two vinyl groups per molecule of EDMA. The compositional drift observed for SPE and HEMA with increasing $f_{1}^{0}$ means that the copolymers produced consist of cross-linked P (SPE-coHEMA) and increasing quantity of PSPE with increasing $f_{1}^{0}$. The PSPE segments could be slightly cross-linked by $\gamma$-irradiation and by reaction with pendant vinyl groups. Consequently, as $f_{1}^{0}$ is increased, the homogeneity of cross-linking diminishes which agrees with the values found for $v_{\mathrm{e}}$ as a function of $f_{1}^{0}$.

Finally, the values of $\phi_{2}$ in conjunction with the values of $v_{\mathrm{t}}$ determined via compression stress-strain experiments and eq 5 allow one

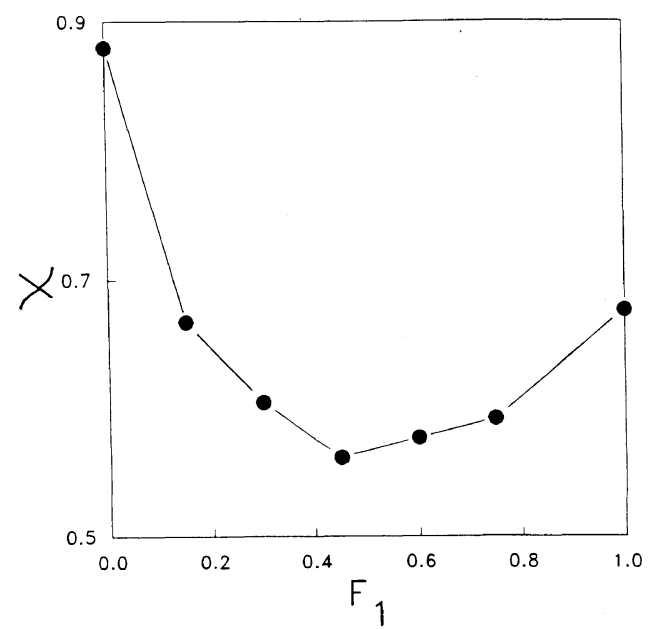

Figure 8. Variation of the interaction parameter $\chi$ with $F_{1}$ for crosslinked SPE/HEMA hydrogels. to determine the interaction parameter $\chi$. It should be noted that the interaction parameters obtained by means of eq 5 do not contain any electrostatic term accounting for the charge density of the polymer chain. Consequently, the values of $\chi$ given here should be considered only as indicators of the overall interactions among water and the three types of monomer unit, SPE, HEMA and EDMA. Figure 8 shows the variation of $\chi$ with $F_{1}$. This figure indicates that the copolymers of all compositions have a greater affinity for water than is exhibited by PHEMA. The minimum value of $\chi$ occurs within the range $F_{1}=0.45-0.75$ which coincides with the range of copolymer composition in which the synergistic swelling takes place. The existence of a minimum value of $\chi(0.56)$ shows that the affinity for water is not simply dictated by increasing content of hydrophilic comonomer but depends on the interaction between SPE nearest neighbours. The novelty and interest of compolymeric hydrogels containing sulphobetaine groups is thereby reinforced.

Acknowledgments. One of us, J.M.R., thanks the Ministerio de Educación y Ciencia of the Spanish Government for the provision of a maintenance grant.

\section{REFERENCES}

1. M. B. Huglin and J. M. Rego, Polymer, in press (1991).

2. M. B. Huglin and J. M. Rego, Macromolecules, 24, 2556 (1991).

3. B. D. Ratner and A. S. Hoffman, "Hydrogels for Medical and Related Applications," J. D. Andrade, A.C.S. Symposium Series 31, Washington D.C., 1976, Chapter 1.

4. M. B. Huglin and D. J. Sloan, Angew Makromol. Chem., 118, 197 (1983).

5. M. B. Huglin and D. J. Sloan, Br. Polym. J., 15, 165 (1983).

6. V. Kudela, in "Hydrogels," Encylopedia of Polymer Science and Engineering," Vol. 7, John Wiley, New York, N. Y., 1988, pp 783-807.

7. T. P. Davis, Doctoral Thesis, University of Salford (1987). 
8. M. Fineman and S. D. Ross, J. Polym. Sci., 5, 259 (1950).

9. D. N. Schulz, D. G. Peiffer, P. K. Agarwal, J. Larabee, J. J. Kaladas, L. Soni, B. Handwerker, and R. T. Garner, Polymer, 27, 1734 (1983).

10. T. P. Davis, M. B. Huglin, and D. C. F. Yip, Polymer, 29, 701 (1988).

11. M. A. Al-Issa, T. P. Davis, M. B. Huglin, J. M. Rego, M. M. M.-A. Rehab, D. C. F. Yip, and M. B. Zakaria, Makromol. Chem., 191, 321 (1990).

12. J. M. Rego, D. M. Bloor, and M. B. Huglin, Polymer, in press (1991).
13. P. J. Flory, "Principles of Polymer Chemistry," Cornell University Press, Ithaca, N.Y., 1953, Chapter XIII.

14. M. B. Huglin, M. M. A.-M. Rehab, and M. B. Zakaria, Macromolecules, 19, 2986 (1986).

15. T. P. Davis and M. B. Huglin, Macromolecules, 22, 2824 (1989).

16. M. B. Huglin and J. M. Rego, Polymer Commun., 32, 129 (1991).

17. "Polymer Handbook," J. Brandrup and E. H. Immergut Ed.), 2nd ed, Wiley, New York, N.Y., 1975, Chapter II, p 387. 